\title{
Promoting Active Living and Healthy Eating among Inner-City Youth through Community Health Workers: From Clinic to Neighborhood
}

\author{
Mihail Subtirelu', Marielisa Rincon-Subtirelu', Michele Pickett ${ }^{2}$, Gregory W. Heath ${ }^{3,4^{*}}$ \\ ${ }^{1}$ Department of Pediatrics, University of Tennessee College of Medicine Chattanooga (UTCOMC), Chattanooga, \\ Tennessee, USA \\ ${ }^{2}$ LifeSpring Community Health, Chattanooga, Tennessee, USA \\ ${ }^{3}$ Department of Medicine, UTCOMC, Chattanooga, Tennessee, USA \\ ${ }^{4}$ University of Tennessee at Chattanooga, Chattanooga, Tennessee, USA \\ Email: ${ }^{*}$ Gregory-Heath@utc.edu
}

Received 22 July 2014; revised 8 September 2014; accepted 22 September 2014

Copyright (C) 2014 by authors and Scientific Research Publishing Inc.

This work is licensed under the Creative Commons Attribution International License (CC BY).

http://creativecommons.org/licenses/by/4.0/

(c) (i) Open Access

\section{Abstract}

The promotion of physical activity and healthy eating to prevent obesity among youth is a pressing challenge. The current study examined the feasibility of community health workers (CHWs) conducting a physical activity (PA) and healthy eating intervention strategy with links to community supports and programs. Youth aged 10 - 18 years were recruited from three clinical sites serving inner-city families. Trained CHWs conducted assessment and counseling for PA and healthy eating among youth and their families and provided customized plans and navigation to neighborhood PA and nutrition programs. Measures of daily PA by self-report, weekday and weekend day sedentary behaviors, fruit and vegetable intake, avoidance of fatty foods, and avoidance of sugary drinks were assessed at baseline and follow-up. Twenty-five patients (mean age = 12.9 years) were exposed to $\sim 9$ months of intervention from baseline. Pre- and post-assessments revealed significant changes in reported PA, sedentary behaviors on weekdays, sedentary behaviors on weekend days, fruit and vegetable intake, avoidance of fatty foods, and avoidance of sugary drinks. Results demonstrated the feasibility of having CHWs effectively influenced the PA and eating behaviors of inner-city youth. Greater success was evident when assessment and counseling for PA and healthy eating were accompanied by navigation to neighborhood resources. The use of CHWs may be a cost-effective approach impacting the PA, sedentary, and dietary behaviors of youth.

\footnotetext{
*Corresponding author.
} 


\title{
Keywords
}

\author{
Physical Activity, Healthy Eating, Community Health Promotion, Community Health Workers, \\ Obesity, Prevention
}

\section{Introduction}

The important influence of physical activity (PA) on reducing the burden of chronic diseases and enhancing quality of life is well established [1] [2]. Diet and PA have been identified as risk factors for overweight, obesity, and type 2 diabetes mellitus (DM) among children, adolescents, and adults [3]. Helping children/youth and their families makes and maintains changes in diet and PA is a pressing challenge. Translation and dissemination of effective behavior change programs to real-world settings is an area requiring additional research [4]. Community-based research methods offer one way to tailor evidence-based interventions to specific community settings [5] [6]. To date, efforts to combine clinic-based preventive care efforts with community-based programming among children and adolescents have not been carried out systematically. Recommendations from the United States Preventive Services Task Force's (USPSTF) Guide to Clinical Preventive Services (the Clinical Guide), which includes recommendations for physical activity and dietary counseling [7], and the physical activity promotion recommendations from The Guide to Community Preventive Services (the Community Guide) [8] [9], along with evidence-based assessment and counseling protocols for youth, referred to as the Patient-centered Assessment and Counseling for Exercise Plus Nutrition materials (PACE+) [10]-[13] offer the substance of an integrated intervention approach. We evaluated the feasibility and use of such evidence-based physical activity and dietary assessment and counseling protocols delivered among low-income, predominantly African-American and Latino pediatric patients (ages 10 - 17 years) attending local community health clinics through the use of locally recruited and trained community health workers (CHWs). It was hypothesized that the combined evidence-based clinical (PACE+) and community-based interventions (local parks and recreation, faith-based, and community programs) would succeed in effectively promoting healthy eating and PA among an at-risk youth population when delivered through the use of trained CHWs.

\section{Methods}

This nine month feasibility project used a multi-component translational intervention study design and focused on primary prevention of the behavioral metabolic disease risk factors of poor dietary habits, physical inactivity, sedentary behaviors, and access to primary health care. The primary intervention sites were a community-based primary care pediatric clinic, and two high-risk pediatric subspecialty practices associated with an academic health center. These three sites are staffed by the academic health center's affiliated faculty. The clinic-based portion of the intervention consisted of assessment and counseling protocols designed to determine youth PA and eating behaviors and to provide customized PA and dietary recommendations.

\subsection{Study Subjects}

The targeted behaviors of PA and healthy eating were directed towards a total of 70 boys and girls who were 10 through 18 years of age along with their families and lived and received their education and health care in several lower income inner-city areas of the Chattanooga, Tennessee region of the United States. Trained CHWs administered all physical activity and dietary assessment tools and provided summary information to primary care providers responsible for each patient (Figure 1). Measures of daily PA were expressed in number of days in the past week in which the respondent engaged in 60 minutes of moderate to vigorous intensity PA (PACE ${ }^{\odot}$ physical activity 7 point scale, 0 days thru 7 days); weekday and weekend day sedentary behaviors (e.g., watching $\mathrm{TV}$, playing video games, computer time, expressed in hours per day); fruit and vegetable consumption (number of servings of fruits and vegetables consumed each day, response categories from $0,1,2,3,4,5$, and $6+$ reported as an average per day); avoidance of fatty foods ( 5 point scale, $1=$ no, and do not intend to in the next 6 months, $2=$ no, but intend to in the next 6 months, $3=$ no, but intend to in the next 30 days, $4=$ yes, but less than 6 months, $5=$ yes, greater than 6 months), and avoidance of sugary drinks ( 5 point scale, $1=$ no, and do not intend to in the next 6 months, $2=$ no, but intend to in the next 6 months, $3=$ no, but intend to in the next 30 days, $4=$ 


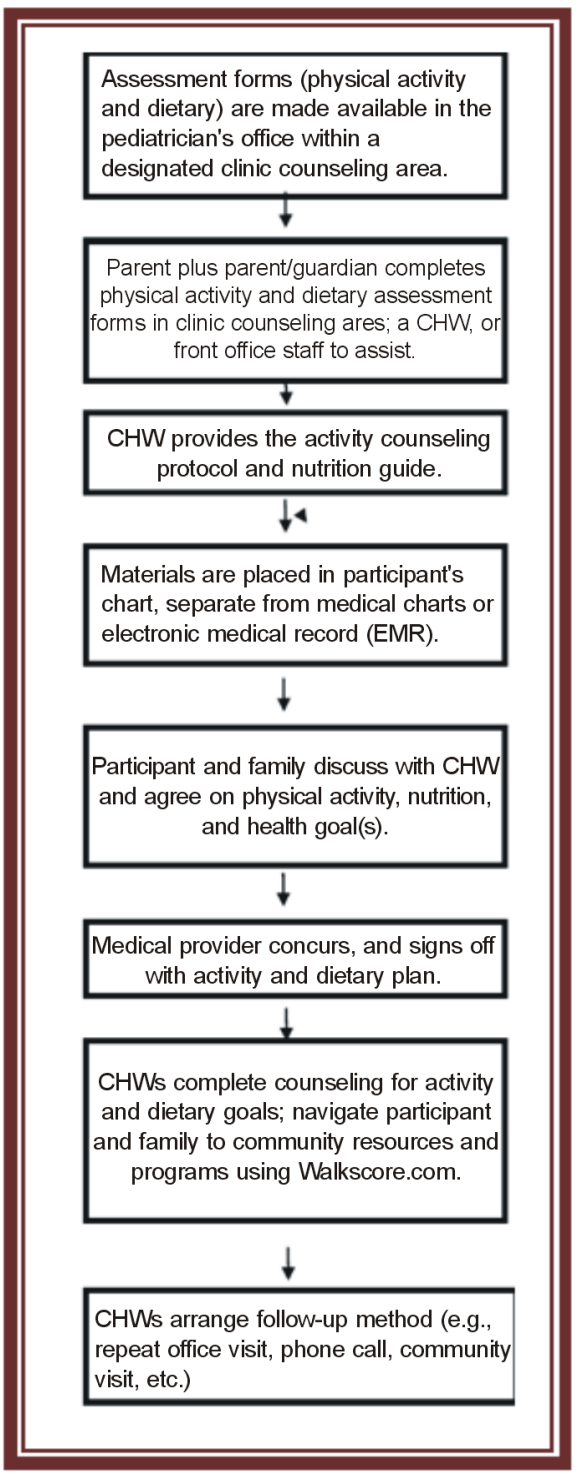

Figure 1. Assessment and counseling flow chart.

yes, but less than 6 months, 5 = yes, greater than 6 months). These health behaviors were assessed at baseline and approximately $\sim 9$ months at follow-up. Each patient's initial assessment and counseling session conducted by the CHWs provided navigational information about environmental and program supports for physical activity and healthy eating in community neighborhoods. The CHWs used a commercially available online tool, Walkscore.com ${ }^{\mathrm{TM}}$, to accomplish this goal. Through the use of this tool, the CHWs provided walking maps, directions to fitness facilities and programs for children and adolescents, healthy eating establishments and markets to all patients undergoing the assessment and counseling.

\subsection{Intervention Strategies}

Intervention strategies included navigating participants to physical activity and nutrition programs offered through local community organizations, for example, local schools, local parks and recreation facilities, faithbased organizations, and other voluntary health organizations. As a result, each patient's physical activity and dietary plan, as detailed in the assessment program by the CHW, was tailored to individual patients not only by using personal behavioral characteristics and strategies, but also by utilizing place of residence, neighborhood 
resources, and access to facilities, affordability of programming, and other supportive environmental moderators.

\subsection{Behavioral and Clinical Data Collection}

Each child's weight and calculated body mass index (BMI) was assessed at baseline and follow-up using clinic charts and/or an electronic health record. Self-reported sedentary behavior (e.g., TV watching, computer gaming, and video watching), physical activity, fruit and vegetable intake, avoidance of fatty foods, and avoidance of sugary drinks was also assessed at baseline and periodically throughout the follow-up period. Human subjects' protection: All patients and their families were provided information about the study. Among families agreeing to participation, adults and children/youth provided informed consent/ascent, respectively. Study protocols and informed/ascent consent forms were approved by the University of Tennessee: College of Medicine Chattanooga/Erlanger Health System Institutional Review Board.

\subsection{Data Analysis}

All clinical and health behavior data were entered into an Excel spreadsheet and kept secure either in a password protected laptop and/or flash drive. Data were imported into IBM SPSS version 21 for data analysis. Summary statistics, means, standard deviation, standard error were calculated for both baseline and follow-up measures. Paired $t$ tests were conducted among all pairs of patient data-(baseline, follow-up). Stratification of data by age and sex were also conducted. Comparisons of baseline measures of age, weight, and BMI among patients completing the intervention trial with 45 patients who elected not to participate in the intervention were conducted and were shown to differ in terms of weight, BMI, and health behaviors (results not shown).

\section{Results}

The mean period of follow-up was 8.6 months and was marked by a repeating of the behavioral assessments, anthropometric and usual care biomedical measures when available. Baseline patient characteristics for 25 patients who participated in the 9 month intervention program are displayed in Table 1. Forty-five youth and their families elected not to participate in the intervention. These subjects were older (mean age $=15$ years), more likely to be Caucasian, had a higher BMI, and lived in more remote areas of the city (data not shown). The mean age among participants was 12.9 years, $68 \%$ were female, with $76 \%$ of the youth of Latino/Hispanic origin and 16\% African-American. Examining the clinical and behavioral measures (Table 1), the initial mean weight of participants was $51.73 \mathrm{Kg}$, while the initial BMI was $23.34 \mathrm{Kg} / \mathrm{m}^{2}$ which represented a mean BMI at the $69^{\text {th }}$ percentile, and a range from the $3^{\text {rd }}$ through the $98^{\text {th }}$ percentile. At follow-up (mean 8.6 months), comparing baseline assessments (Figure 2) revealed significant changes in reported physical activity(baseline 3.34 vs. follow-up 5.72, p < 0.0001); sedentary behaviors on weekdays (baseline $4.5 \mathrm{hr} /$ day vs. follow-up $2.1 \mathrm{hr} / \mathrm{day}$ ); sedentary behaviors on weekend days (baseline $3.5 \mathrm{hr} /$ day vs. follow-up $2.6 \mathrm{hr} /$ day, $\mathrm{p}<0.001$ ); fruit and vegetable intake (baseline 3.1 servings per day vs. follow-up 5.0 servings per day, $\mathrm{p}<0.0001$ ); avoidance of fatty foods (baseline 2.95 vs. follow-up 4.8, p < 0.001); and avoidance of sugary drinks (baseline 3.05 vs. follow-up 4.20, p $<0.001$ ). There were no significant changes in weight (51.72 kg vs. $52.22 \mathrm{~kg}$ ) or BMI (23.34 vs. 23.30) noted during the observation period.

Table 1. Participant characteristics at baseline $(n=25)$.

\begin{tabular}{ccc}
\hline Characteristic & Mean & Standard Deviation \pm \\
Age (years) & 12.9 & 2.85 \\
Weight $(\mathrm{Kg})$ & 51.72 & 21.12 \\
BMI $\left(\mathrm{Kg} / \mathrm{m}^{2}\right)$ & 23.34 & Proportion (\%) \\
\hline Female & Number & $60 \%$ \\
Male & 17 & $40 \%$ \\
Latino/Hispanic Race/Ethnicity & 8 & $76 \%$ \\
African-American Race/Ethnicity & 19 & $16 \%$ \\
Caucasian/White Race/Ethnicity & 4 & $8 \%$ \\
\hline
\end{tabular}




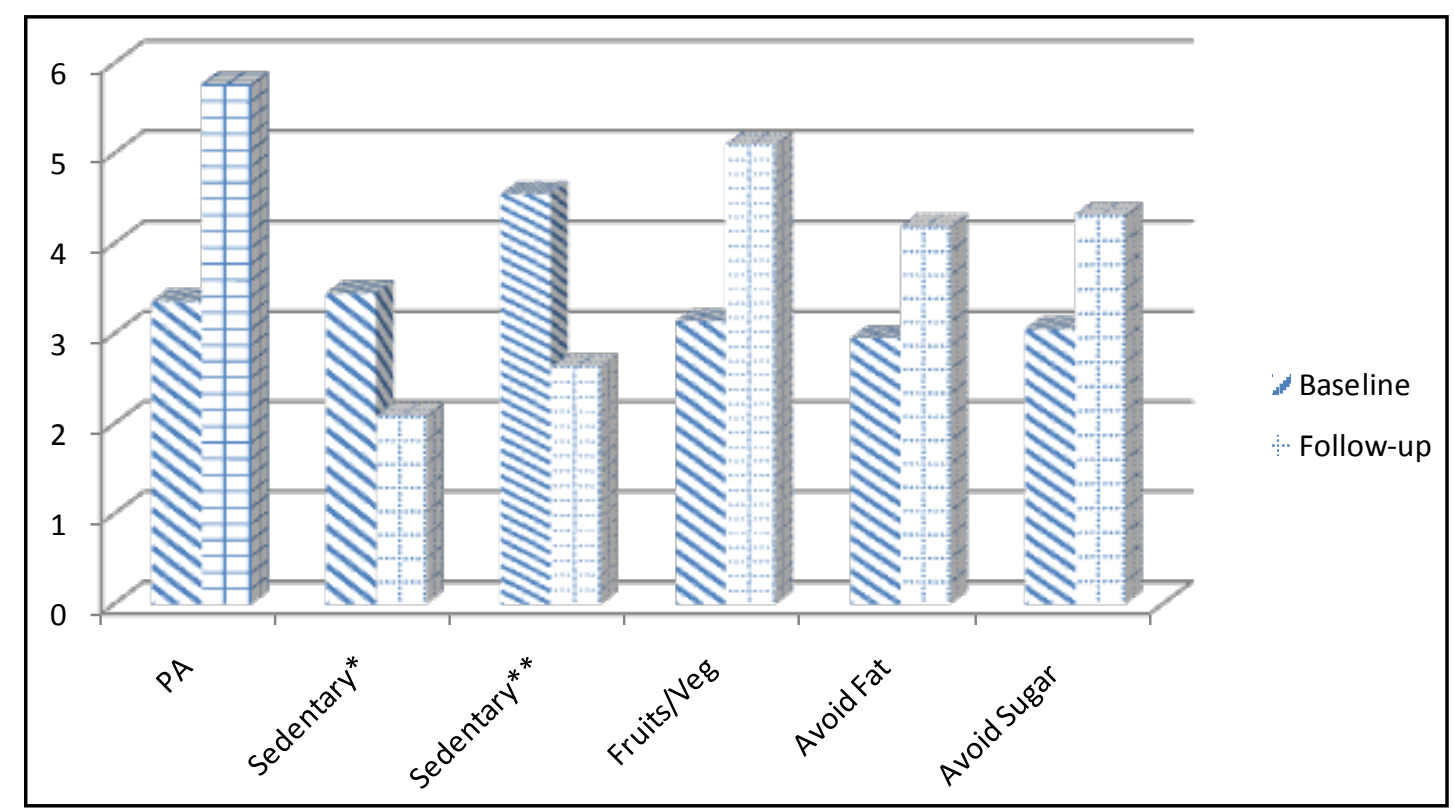

Figure 2. Baseline and follow-up health behavior measures $(n=25)$. (All comparisons are significant, using paired t tests@ $\mathrm{p}<0.001)$. PA = Days per week of 60 or more minutes per day of moderate to vigorous physical activity; Sedentary $^{*}=$ Number of hours per day of sedentary activities on week days; Sedentary ${ }^{* *}=$ Number of hours per day of sedentary activities on weekend days; Fruits/Veg = Number of fruits and vegetables consumed per day during the past 7 days; Avoid fat = Avoid fatty foods scale; $(1=$ No; 2 = No, but intend to in 6 months; 3 = No, but intend to in 3 months; 4 = Yes, have but less than 6 months; 5 = Yes, have for more than 6 months); Avoid sugar = Avoid sugary drinks (soda and juices) scale; $(1=$ No; 2 = No, but intend to in 6 months; $3=$ No, but intend to in 3 months; 4 = Yes, have but less than 6 months; 5 = Yes, have for more than 6 months).

\section{Discussion}

The results from this feasibility study suggest that trained CHWs can influence the physical activity and eating behaviors of inner-city youth when assessment and counseling for physical activity and healthy eating is accompanied by navigation to neighborhood resources. The use of CHWs may be a cost-effective approach impacting the physical activity, sedentary, and dietary behaviors of youth. Our results appear to be quite timely since the rate of overweight and obese children and adolescents in the United States continues to increase. The increase in the prevalence of overweight and obesity among children and adolescents is similar to estimates among adults, and has been increasing disproportionately among African-American and Latino children and adolescents. Sixteen percent (16\%) of African-American children are overweight, and an additional 15\% are at risk for overweight or becoming obese. Latino children are the most overweight ethnic group of US children, with $22 \%$ overweight and $40 \%$ at risk for overweight or obesity, compared with $14 \%$ and $28 \%$ for white children, and $21 \%$ and $35 \%$ for African-American children, respectively [14]. Similar findings exist among the children and youth living in the region from which the subjects of the current study are drawn [15]. The advantages of modern technologies have fostered an epidemic of sedentary lifestyles and reduced levels of physical activity, with only $60 \%$ of American adolescents active at levels recommended for achieving health benefits, with nearly $25 \%$ of adolescents being completely sedentary outside of school [16]. Within the current study at baseline only $10 \%$ of youth were meeting the Physical Activity Guidelines for Americans, of 60 minutes of moderate to vigorous PA per day. Effective community-based strategies for physical activity promotion exist and are beginning to be utilized as noted in the current study. These evidence-based strategies have been reviewed by the Task Force on Community Preventive Services [8]. The use of CHWs evidenced in the present study underscores their application and use in the pediatric healthcare setting. Since CHWs are most often local community volunteers, as was the case in our study, these personnel are key people in their respective communities [17]. These individuals were selected and trained to provide counseling and guidance for active living and healthy eating among clinic patients and their families. The CHWs worked closely with clinic staff to ensure fidelity with each of the evidence-based protocols for healthy eating and active living used both in the clinic and 
community settings. Using CHWs for patient navigation is a key innovative element in our project. Our results are similar to the initial findings of Patrick, et al., [10] where they employed community health educators and health behavior specialists. Hence the role of the CHWs in the present study clearly helped the research team accomplish the primary aims of the study in terms of positive health behavior change. The patient navigation component of the study helped ensure that patients and their families were made aware of and are put in contact with community structures, facilities, and programming in a practical and useful manner. The present study is one of the first to show the efficacy of using CHWs to implement successful active living and healthy eating interventions among a pediatric population as well as adding to growing literature that demonstrates the effective use of CHWs among persons living in low income communities to promote health and disease management strategies [18]-[20]. Our study is subject to the limitations of participants serving as their own controls; hence issues of self-selection and generalizability may exist. Finally, although all health behavior assessments were conducted by trained CHWs in person using valid and reliable instruments, the results are based on self-reported information and may be subject to some level of ascertainment bias.

\section{Conclusion}

Our study demonstrated that the innovative use of enhanced follow-up methods, such as implementing a patient navigation system that directs participants to environmental supports within their communities for active living and healthy eating, increasing the likelihood of translating and sustaining these important health behaviors which may be linked to the effective prevention and management of obesity and associated co-morbidities.

\section{Acknowledgements}

We wish to thank our Community Health Workers, Eunice Mendoza and Elizabeth Tenley, for their diligent and faithful work among our patients, their families, and community partners.

\section{References}

[1] Haskell, W.L., Lee, I.-M., Pate, R.R., Powell, K.E., Blair, S.N., Franklin, B.A., Macera, C.A., Heath, G.W., Thompson, P.D. and Bauman, A. (2007) Physical Activity and Public Health: Updated Recommendation for Adults from the American College of Sports Medicine and the American Heart Association. Circulation, 116, 01-13.

[2] Physical Activity Guidelines Advisory Committee (2008) Physical Activity Guidelines Advisory Committee Report, 2008. U.S. Department of Health and Human Services, Washington, DC.

[3] McGinnis, J.M. and Foege, W.H. (1993) Actual Causes of Death in the United States. JAMA, 270, 2207-2212. http://dx.doi.org/10.1001/jama.1993.03510180077038

[4] Green, L.W. and Johnson, J.L. (1996) Dissemination and Utilization of Health Promotion and Disease Prevention Knowledge: Theory, Research and Experience. Canadian Journal of Public Health, 87, S211-S217.

[5] Eyler, A.A., Mayer, J., Rafii, R., Housemann, R., Brownson, R.C. and King, A.C. (1999) Key Informant Surveys as a Tool to Implement and Evaluate Physical Activity Interventions in the Community. Health Education Research, 14, 289-298. http://dx.doi.org/10.1093/her/14.2.289

[6] Haire-Joshu, D., Brownson, R.C., Schechtman, K., Nanney, S., Houston, C. and Auslander, W. (2001) A Community Research Partnership to Improve the Diet of African Americans. American Journal of Health Behavior, 22, 140-146. http://dx.doi.org/10.5993/AJHB.25.2.6

[7] Harris, R.P., Helfand, M., Woolf, S.H., et al. (2001) Current Methods of the U.S. Preventive Services Task Force. A Review of the Process. American Journal of Preventive Medicine, 20, 21-35. http://dx.doi.org/10.1016/S0749-3797(01)00261-6

[8] Kahn, E.B., Ramsey, L.T., Brownson, R.C., et al. (2002) The Effectiveness of Interventions to Increase Physical Activity: A Systematic Review. American Journal of Preventive Medicine, 22, 73-107. http://dx.doi.org/10.1016/S0749-3797(02)00434-8

[9] Heath, G.W., Brownson, R.C., Kruger, J., et al. (2006) The Effectiveness of Urban Design and Land Use and Transport Policies and Practices to Increase Physical Activity: A Systematic Review. Journal of Physical Activity and Health, 3, S55-S76.

[10] Patrick, K., Calfas, K.J., Norman, G.J., Zabinski, M.F., Sallis, J.F., Rupp, J., Covin, J. and Cella, J. (2006) Randomized Controlled Trial of a Primary Care and Home-Based Intervention for Physical Activity and Nutrition Behaviors: PACE+ for Adolescents. Archives of Pediatrics and Adolescent Medicine, 160, 128-136. 
[11] Long, B.J., Calfas, K.J., Patrick, K., Sallis, J.F., Wooten, W.J., Goldstein, M., Marcus, B., Schwenk, T., Carter, R., Torrez, T., Polinkas, L. and Heath, G. (1996) A Multisite Field Test of the Acceptability of Physical Activity Counseling in Primary Care: Project PACE. American Journal of Preventive Medicine, 12, 73-81.

[12] Calfas, K.J., Long, B.J., Sallis, J.F., Wooten, W.J., Pratt, M. and Patrick, K. (1996) A Controlled Trial of Physician Counseling to Promote the Adoption of Physical Activity. Preventive Medicine, 25, 225-233. http://dx.doi.org/10.1006/pmed.1996.0050

[13] Calfas, K.J., Sallis, J.F., Oldenburg, B. and French, M. (1997) Mediators of Change in Physical Activity Following an Intervention in Primary Care: PACE. Preventive Medicine, 26, 297-304. http://dx.doi.org/10.1006/pmed.1997.0141

[14] Ogden, C.L., Carroll, M.D., Curtin, L.R., Lamb, M.M. and Flegal, K.M. (2010) Prevalence of High Body Mass Index in US Children and Adolescents, 2007-2008. JAMA, 303, 242-249. http://dx.doi.org/10.1001/jama.2009.2012

[15] Centers for Disease Control and Prevention/National Center for Chronic Disease Prevention and Health Promotion/ Division of Nutrition, Physical Activity, and Obesity (2012) Tennessee: State Nutrition, Physical Activity, and Obesity Profile. http://www.cdc.gov/obesity/stateprograms/fundedstates/pdf/tennessee-state-profile.pdf

[16] Mokdad, A.H., Bowman, B.A., Ford, E.S., Vinicor, F., Marks, J.S. and Koplan, J.P. (2001) The Continuing Epidemics of Obesity and Diabetes in the United States. JAMA, 286, 1195-1200. http://dx.doi.org/10.1001/jama.286.10.1195

[17] Brownstein, N.J., Bone, L.R., Dennison, C.R., Hill, M.N., Kim, M.T. and Levine, D.M. (2005) Community Health Workers as Interventionists in the Prevention and Control of Heart Disease and Stroke. American Journal of Preventive Medicine, 29, 128-133. http://dx.doi.org/10.1016/j.amepre.2005.07.024

[18] Walker, D.G. and Jan, S. (2005) How Do We Determine Whether Community Health Workers Are Cost-Effective? Some Core Methodological Issues. Journal of Community Health, 30, 221-229. http://dx.doi.org/10.1007/s10900-004-1960-4

[19] Norris, S.L., Chowdhury, F.M., Van Le, K., Horsley, T., Brownstein, J.N., Zhang, X., et al. (2006) Effectiveness of Community Health Workers in the Care of Persons with Diabetes. Diabetic Medicine, 23, 544-556. http://dx.doi.org/10.1111/j.1464-5491.2006.01845.x

[20] Ingram, M., Torres, E., Redondo, F., Bradford, G., Wang, C. and O’Toole, M. (2007) The Impact of Promotoras on Social Support and Glycemic Control among Members of a Farmworker Community on the US-Mexico Border. The Diabetes Educator, 33, 172S-178S. 
Scientific Research Publishing (SCIRP) is one of the largest Open Access journal publishers. It is currently publishing more than 200 open access, online, peer-reviewed journals covering a wide range of academic disciplines. SCIRP serves the worldwide academic communities and contributes to the progress and application of science with its publication.

Other selected journals from SCIRP are listed as below. Submit your manuscript to us via either submit@scirp.org or Online Submission Portal.
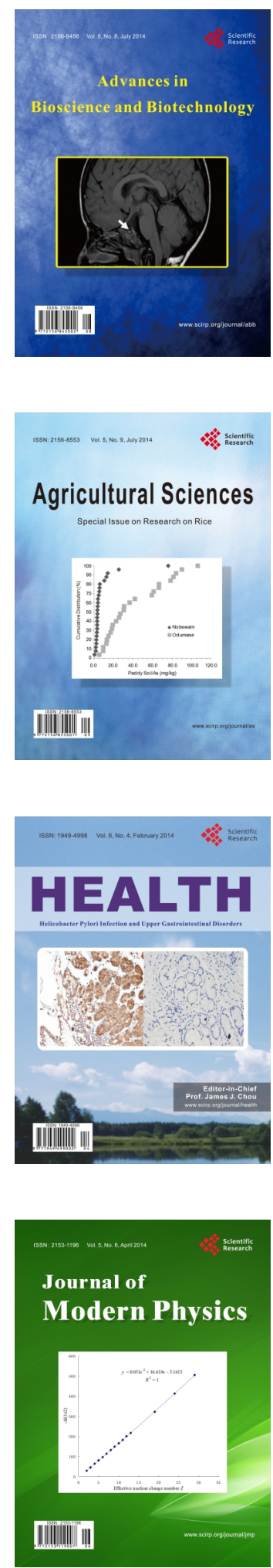
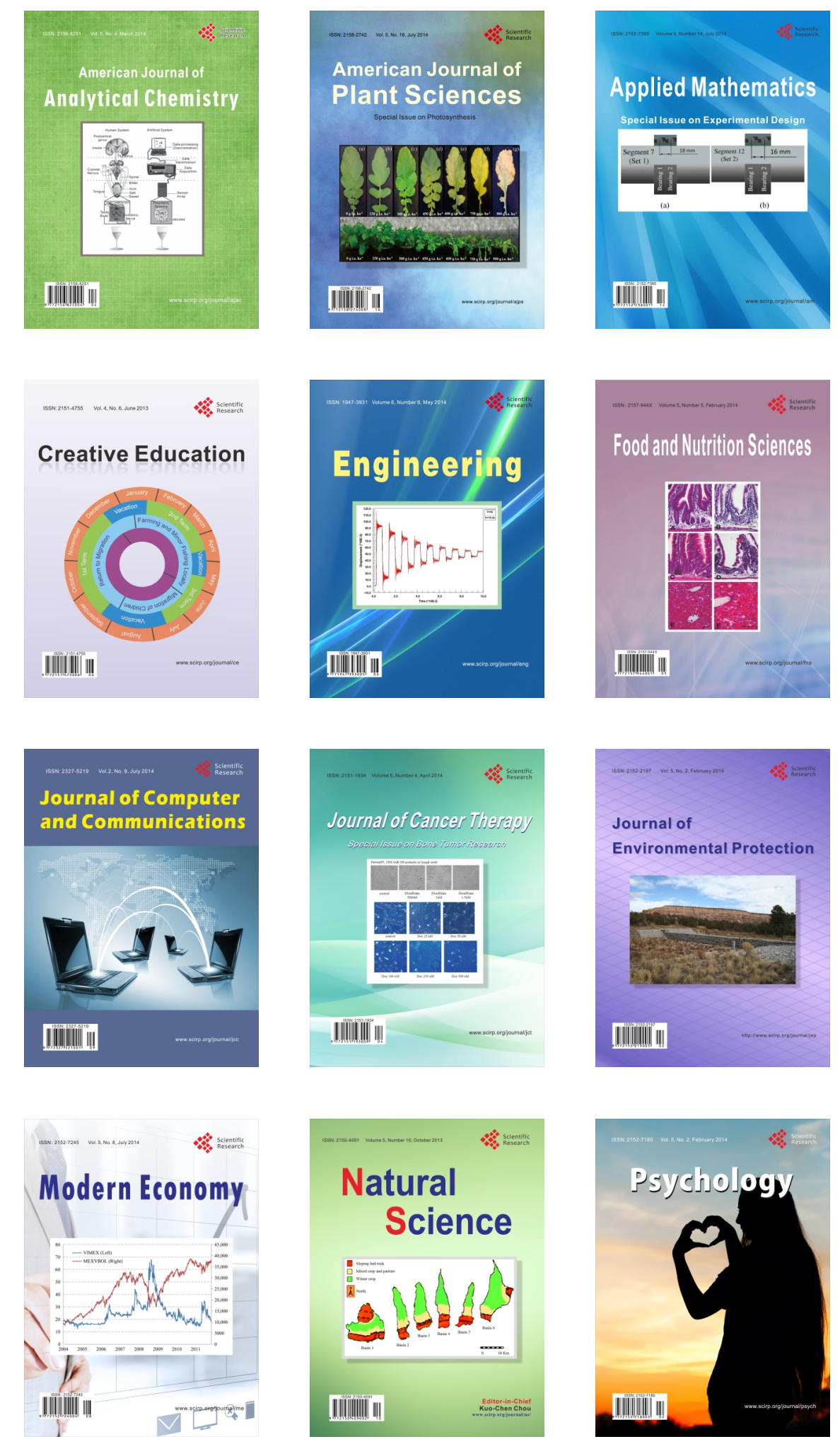